\title{
Structural and energetic properties of canonical and oxidized telomeric complexes studied by molecular dynamics simulations
}

\author{
Przemysław Czeleń • Piotr Cysewski
}

Received: 20 December 2012 / Accepted: 17 April 2013 / Published online: 15 May 2013

(C) The Author(s) 2013. This article is published with open access at Springerlink.com

\begin{abstract}
The structural and energetic properties of native and oxidized telomeric complexes were defined by means of molecular dynamic (MD) simulations. As a starting point, the experimental conformation of B-DNA d(GpTpTpApG pGpGpTpTpApGpGpG) oligomer bound to human protein telomeric repeat binding factor 1 (TRF1) was used. The influence on the stability of the telomeric complex of the presence of 8-oxoguanine (8oxoG) in the central telomeric triad (CTT) was estimated based on trajectories collected during $130 \mathrm{~ns}$ MD runs. The data obtained indicate that the system analyzed is highly sensitive to the presence of oxidative damage in the CTT of the B-DNA telomeric sequence. The most important changes were observed in the immediate vicinity of the 8-oxoguanine molecule. The significantly higher mobility of arginine 425 interacting directly with the oxidized guanine molecule has a large influence on the structural, dynamic and energetic properties of neighboring amino acids. Local changes observed for individual hydrogen bonded interactions localized in the major groove of B-DNA also have significant impact on the properties of hydrophobic clusters, which are the second type of force responsible for stability of the studied bio-system. All the changes reported in detail here unambiguously indicate a significant decrease in telomer binding affinity after oxidation.
\end{abstract}

Electronic supplementary material The online version of this article (doi:10.1007/s00894-013-1859-z) contains supplementary material, which is available to authorized users.

P. Czeleń $(\bowtie) \cdot$ P. Cysewski

Physical Chemistry Department, Collegium Medicum

in Bydgoszcz, Nicolaus Copernicus University in Toruń,

Kurpińskiego 5, 85-950 Bydgoszcz, Poland

e-mail: przemekcz@cm.umk.pl

P. Cysewski

Faculty of Chemical Technology and Engineering,

University of Technology and Life Sciences,

Seminaryjna 3, 85-326 Bydgoszcz, Poland
Keywords Telomers - TRF1 - Oxidative damage · 8-oxoguanine $\cdot$ Molecular dynamics

\section{Introduction}

A key aspect of all living organisms is the proper preservation of genetic material. Indeed, very similar systems for protection of genome stability exist in all organisms, from bacteria to humans. One such system involves telomeres - specific biocomplexes formed between telomeric DNA sequences and dedicated proteins [1-8] that are found at the ends of chromosomes in every living cell $[9,10]$. Telomeres protect the termini of B-DNA from being recognized as B-DNA double-strand breaks [11]. The progressive shortening of telomeric DNA that occurs during replication processes is one of the most important factors regulating cellular lifetime; therefore, telomeres are often termed the "mitotic clock" [12-14]. Dysfunction of these structures can lead to fusion of chromosome ends, acceleration of senescence, or even apoptosis [15-18].

Numerous sources indicate that telomere instability can be related to accumulation of damage imposed by the activity of reactive oxygen forms. The characteristic repetitive telomeric sequence (AGGGTT) $[19,20]$ - with three guanine molecules in the central telomeric triad (CTT) - exhibits greater susceptibility to oxidation than other DNA fragments [21]. This is related to strong experimental evidence that guanine is the most probable site for hole trapping [22, 23], since it has the smallest ionization potential (IP) value of all purines and pyrimidines [24-26]. Moreover, since GG and GGG stacked guanine sequences have lower IPs than single guanines [24, 27], such sequences are even more probable sites for undergoing oxidation. Also, formation of complexes by telomeric sequences with telomeric repeat binding factor (TRF) protein ligands is affected greatly by the presence of oxidative damage in the guanine triad $[28,29]$. The appearance of a single 8-oxoguanine molecule decreases binding affinity between DNA and protein by about $50 \%$, while the presence of 
multiple 8-oxoguanine molecules in the telomeric sequence completely disrupts binding [30-32]. The most important changes related to the presence of the 8-oxoguanine molecule in telomeric sequences are observed in the space of the major groove $[28,29,33]$, which plays the role of an active site responsible for binding of protein ligands. Figure 1 presents the structure of single subunit formed by one telomeric sequence and TRF1 protein ligand. The stability of such a system is maintained mainly by two kinds of interactions: hydrogen bonding and hydrophobic interactions. In the main strand of the telomeric sequence, significant contacts between guanine DG6 and arginine ARG425 are observed. Other bases and phosphate groups from side chains also create important connections, e.g., adenine DA4 and guanine DG5 with lysine LYS421 and tryptophan TRP403. The complementary strand is stabilized by the interactions of cytosines DC6 ${ }^{\prime}$ and $\mathrm{DC}^{\prime}{ }^{\prime}$ with aspartic acid ASP 422. Also, the side chains of DC7' and DA8' are stabilized by hydrogen bonds with active groups of arginines ARG423, ARG415 and tryptophan TRP 383. In the space of the major groove, one can also find a hydrophobic cluster formed by methionine MET 419, valine VAL418, cytosines DC7', DC6' and thymine DT3 [34, 35].

Analysis of these interactions and the changes imposed by oxidation of guanine to $8 \mathrm{oxoG}$ can help in understanding the source of experimentally observed reduction of TRF 1 affinity toward B-DNA complex formation. Our previous investigations focused on analysis of the structural and energetic properties of native and oxidized telomeric B-DNA sequences $[28,29]$. The presence of an 8-oxoguanine molecule in CTT was directly related to a significant increase in the stiffness of its conformation. This structural stiffness was manifested mainly by a reduction in opening and rolling of pairs comprising the oxidized guanine. The main source of this phenomenon is the large increase in stacking and hydrogen bond interactions of 8-oxoguanine with neighboring molecules $[29,33]$. Binding of telomeric sequences with TRF1 ligand is the next most important factor responsible for increased stiffness of the B-DNA double helix. This is related to a reduction in twisting and restriction of mutual shifting and sliding of base pairs forming CTT [28]. These big differences in the properties of canonical and oxidized units indicate a significant sensitivity of TRF1 binding to telomeric sequence.

Our previous discussion $[28,29]$ was restricted exclusively to a description of the role of the CTT. Ignoring changes in more extended regions might provide too narrow a perspective. For this reason, we are extending the analysis and taking into account also changes in the vicinity of the CTT. There is another serious reason for re-examining the system formed by TRF1 and B-DNA telomeric subunits. Our previous investigations dealt with very short trajectories. Here, a much longer time evolution is presented, encompassing a 130 -ns time window. Thus, the aim of this study was to extend and broaden the energetic and structural analysis of native and oxidized telomeric B-DNA sequences characterizing in detail the interactions between all monomers involved in formation of binding in the active site. Based on this wider perspective, the direct influence of guanine molecule oxidation on the stability of telomeric complexes is examined. Importantly, instead of just the energy of interaction, both energetic (enthalpic) and entropic contributions are provided.

\section{Methods}

The time evolution of a model system comprising a tridecamer in B-DNA form complexed with the human protein TRF1 was studied based on molecular dynamics (MD) simulations. The geometry of the initial structure was taken from RCSB Protein Data Bank deposit 1IV6.pdb [36]. The protein-DNA complex was immersed in a TIP3P water box $[37,38]$. The same initial structure was used for both canonical (S1) and oxidized (S2) oligonucleotides. The
Fig. 1 Graphical representation of telomeric B-DNA subunit complexed to human telomeric repeat binding factor 1 (TRF1) protein. In the red tetragon is presented simplified arrangement of telomeric B-DNA sequence and localization of the most important amino acids involved in creation of bindings in the space of active site

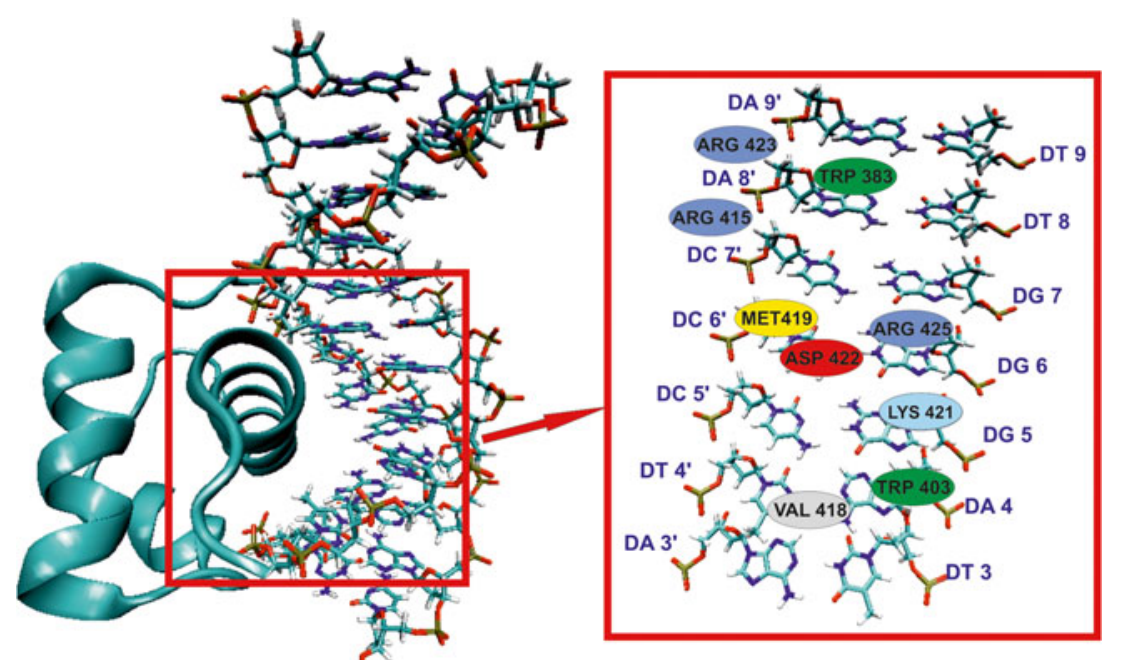


latter corresponds to a telomere oligomer in which the guanine in the central telomeric triad was replaced with (8oxoG), forming a d $\left(\mathrm{G}_{1} \mathrm{pT}_{2} \mathrm{pT}_{3} \mathrm{pA}_{4} \mathrm{pG}_{5} \mathrm{pZ}_{6} \mathrm{pG}_{7} \mathrm{pT}_{8} \mathrm{pT}_{9}\right.$ $\mathrm{pA}_{10} \mathrm{pG}_{11} \mathrm{pG}_{12} \mathrm{pG}_{13}$ ) tridecamer, where $\mathrm{Z}$ stands for $\mathrm{G}$ or 8oxoG. A graphical representation of the complex analyzed is shown in Fig. 1. Charge of the system was neutralized by sodium cations. The analyzed systems were heated to $300 \mathrm{~K}$ during $100 \mathrm{ps}$ of MD simulation and the temperature was controlled by a Langevin thermostat. Periodic boundary conditions and the SHAKE algorithm [39] were applied for 130 ns MD simulations. Initially, a 30-ns interval was used for system equilibration. Equilibration was confirmed by stable fluctuations of density, temperature and energy values. The structural analysis encompassed snapshots taken from the final $100 \mathrm{~ns}$ every $10 \mathrm{ps}$. In this manner, 10,000 conformations were used to determine the characteristics of both native and oxidized systems. The analysis was performed using the VMD package [40]. The molecular mechanic/PoissonBoltzmann surface area (MMPBSA) method [41] was used for free energy computations. Independent calculations were conducted for enthalpic and entropic contributions to Gibbs free energy values. Also, decomposition of enthalpic contributions was performed for selected nucleobases and aminoacids. Enthalpic contribution to Gibbs Free energy was calculated using all the conformations collected during MD simulations. Due to the much higher computation cost of entropic contributions, the population of conformations used was reduced tenfold. This made the computation feasible in a reasonable time. In all MD simulations, the AMBER 11 package [42] was used with the Barcelona corrected forcefield [43] supplemented by parameters for oxidized guanine [44]. For free energy calculations, the AmberTools 1.5 package was used.

\section{Results and discussion}

\section{Chemical affinity of TRF1 for telomeric B-DNA}

Understanding the impact on stability of telomeric complexes of the presence of 8-oxoguanine requires accurate knowledge of the characteristics of the interactions between all considered monomers. In this work, we determined the global and local contributions to stability of native and oxidized systems, which were characterized by Gibbs free energy values. Table 1 presents values for enthalpic and entropic contributions to total extension values of Gibbs free energy of the analyzed system. The data presented clearly suggest that oxidation of CTT affects both $\Delta H$ and $\Delta \mathrm{S}$ contributions. The observed changes in $\Delta \mathrm{G}$ values allow us to conclude that stability of the telomeric complex decreased after oxidation. The oxidized telomere fragment makes a smaller (by ca. $11 \mathrm{kcal} \mathrm{mol}^{-1}$ ) enthalpic contribution to the
Table 1 Value of Gibbs free energy characterizing the stability of native (S1) and oxidized (S2) complexes of telomeric oligonucleotides and TRF1 protein. All values are in $\mathrm{kcal} / \mathrm{mol}$

\begin{tabular}{llll}
\hline Structure & $\Delta \mathrm{H}$ & $\Delta \mathrm{S} \cdot \mathrm{T}$ & $\Delta \mathrm{G}$ \\
\hline S1 & $-128.3 \pm 12.8$ & $-36.1 \pm 8.2$ & $-92.2 \pm 15.2$ \\
S2 & $-117.3 \pm 14.6$ & $-39.6 \pm 5.5$ & $-77.6 \pm 15.6$ \\
\hline
\end{tabular}

affinity of TRF1 for the B-DNA oligomer; application of a $t$-test confirmed that this difference is significant $(P<0.001)$. The averaged entropic contributions to $\Delta \mathrm{G}$ are nearly the same magnitude for both canonical and oxidized structures. It is worth noting that, in this case, the distributions are statistically distinct $(P<0.01)$. The chemical affinity for TRF1 binding to the native telomeric B-DNA sequence is about $15 \mathrm{kcal} \mathrm{mol}^{-1}$ higher compared to that of the oxidized system. Thus, the overall stability of the oxidized system is lower and formation of the nucleic acid complex with TRF1 less efficient.

The time evolution of enthalpic and entropic contributions to Gibbs free energy was also determined. The data plotted in Figs. 2 and 3 indicate unambiguously that the distributions of $\Delta \mathrm{H}$ and $\Delta \mathrm{S}$ values for native and oxidized systems are entirely different. During the simulation, numerous intervals characterized by enthalpy deviations as high as $30 \mathrm{kcal} \mathrm{mol}^{-1}$ were observed. Interestingly, the discrepancy between the entropic contributions to Gibbs free energy of S1 and S2 becomes more significant with longer time of simulation suggesting that the observed trend is rather persistent. The systematic rise in energy up to $10 \mathrm{kcal} \mathrm{mol}^{-1}$ clearly indicates the decrease in stability of the oxidized complex. The observed differences in global values of Gibbs free energy have their origin in the numerous changes reported for individual interactions localized in the space of the major groove.

Table 2 lists the values for enthalpic contributions of selected pairs of interacting molecules. The most significant changes are observed in the close vicinity of oxidative CTT damage. Regarding interactions of ARG 425 with the guanine triad, a comparison of the values for native and oxidized system indicates a decrease in binding affinity of ARG 425 to DZ6 of $4.7 \mathrm{kcal} \mathrm{mol}^{-1}$, and a simultaneous increase (by about $2 \mathrm{kcal} \mathrm{mol}^{-1}$ ) of ARG 425 interactions with DG7 after oxidation. Furthermore, values of standard deviation characterizing the latter interactions are significantly higher, suggesting the existence of many conformations with unfavorable energy values. Very similar effects were also observed for interactions between arginine ARG 423 and nucleotide DA8'. In this case, the observed decrease in energy values is accompanied by increasing standard deviation. Interesting dependencies also occur for the interactions of lysine LYS 419 with nucleotides DA4 and DG5. In the native system, both interactions are characterized by 
Fig. 2 Time evolution of enthalpic contribution $(\Delta \mathrm{H})$ to Gibbs free energy of the studied native and oxidized telomeric complexes

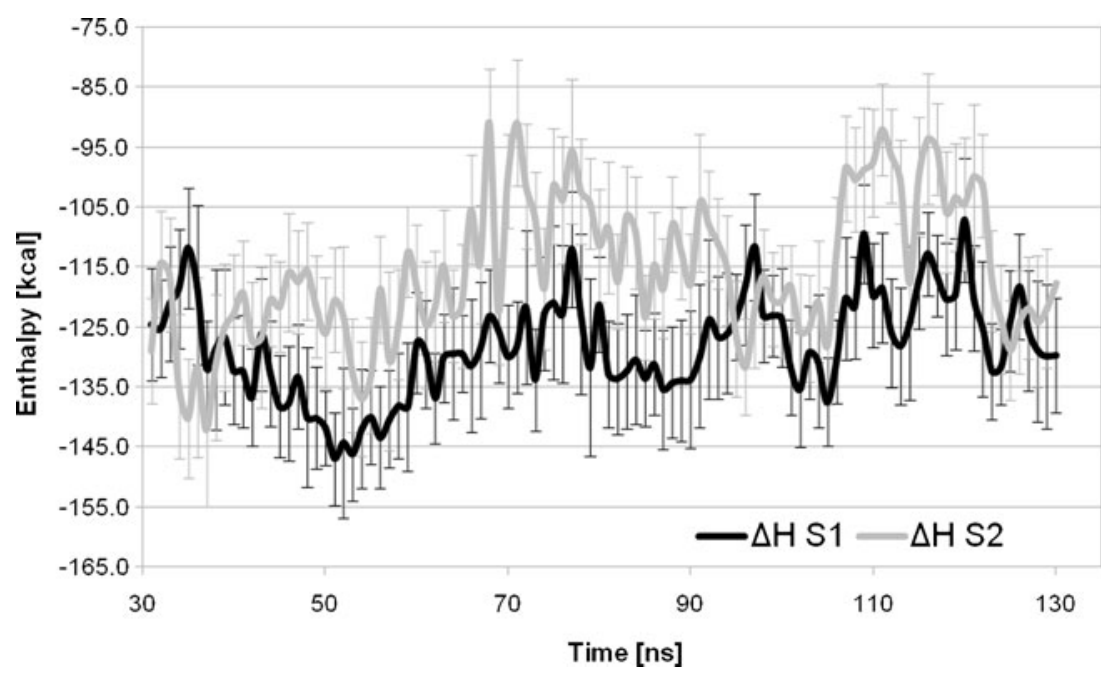

similar values of enthalpic contribution to Gibbs free energy, while in the S2 system a noticeable decrease was observed for LYS 419 and DG5. Additionally, a significant increase in energy was also observed in the interaction with LYS 419 DA4. This confirms not only the rise in energy values compared to the reference system but also, which is equally interesting, the decrease of standard deviation values, suggesting tighter and more uniform binding.

\section{Structural properties of the TRF1-telomere complex}

Trajectories collected during MD simulations were used to determine the quantitative characteristics of all interactions in native and oxidized systems. The mutual orientation of functional groups was evaluated for all interactions localized in the major groove. For hydrogen bonding characteristics, the distances between hydrogen atoms and electronegative atoms $(\mathrm{O}, \mathrm{N})$ were measured, while hydrophobic contacts were identified by measuring distances between carbon atoms of the groups of interest. The dynamic properties of selected amino acids interacting with telomeric sequences were evaluated using RMSD values. The most notable discrepancies were observed in the close vicinity of the 8-oxoguanine molecule. Smoothed histograms illustrating the distribution of hydrogen bonds formed between the active groups of arginine ARG425 and guanine are presented in Fig. 4. These distributions suggest that, for the native structure, both amino groups of arginine form stable hydrogen bonds with oxygen $\mathrm{O} 6$ and nitrogen N7 of guanine DG6 during almost the entire simulation, while simultaneous interactions with other guanine molecules are nearly incidental.

The behavior of the same amino acid in complex with the oxidized telomeric sequence is completely different. The frequency of interactions with 8-oxoguanine is much lower compared to the native system. Capping the nitrogen N7 center with a hydrogen atom in the 8-oxoguanine molecule significantly impedes interactions with the amino groups of arginine ARG425. The hydrogen bonds formed by oxygen
Fig. 3 Distribution of entropic contribution $(\Delta S)$ to Gibbs free energy obtained for every $100 \mathrm{ps}$ from the considered trajectory

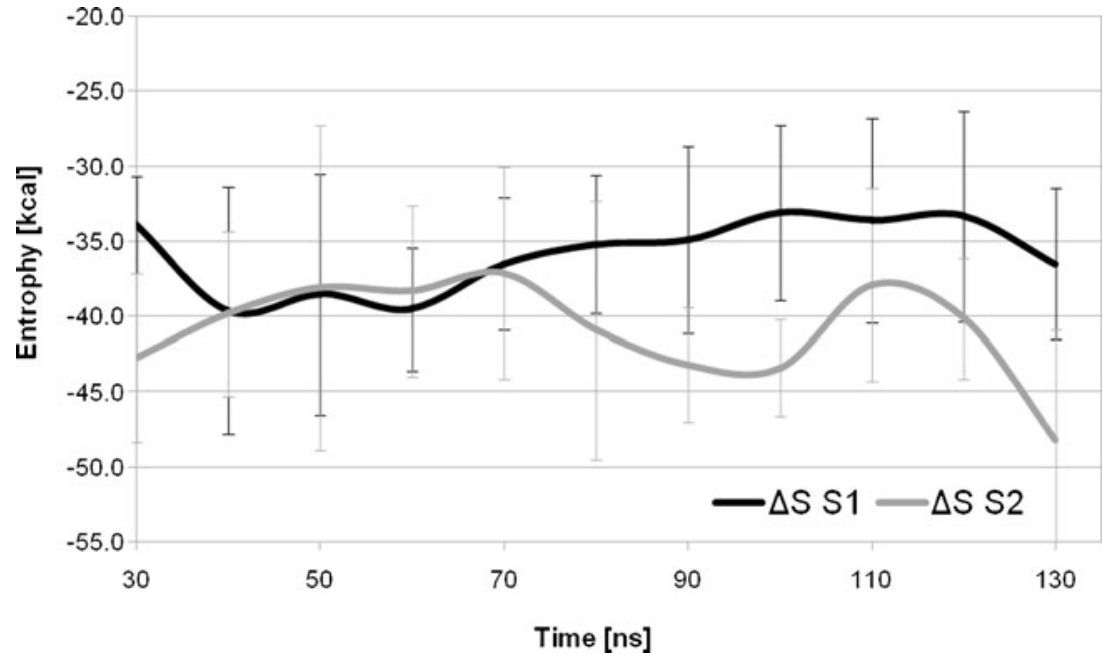


Table 2 Enthalpic contributions to Gibbs free energy values (kcal/ mol) with standard deviation values decomposed into crucial interactions in the space of major groove

\begin{tabular}{|c|c|c|c|}
\hline Residue 1 & Residue 2 & $\begin{array}{l}\mathrm{S} 1 \\
\Delta \mathrm{H}\end{array}$ & $\begin{array}{l}\mathrm{S} 2 \\
\Delta \mathrm{H}\end{array}$ \\
\hline DT 3 & LYS 419 & $-2.1 \pm 1.9$ & $-2.2 \pm 1.1$ \\
\hline DA 4 & LYS 419 & $-10.9 \pm 8.6^{\mathrm{a}}$ & $-11.3 \pm 3.3$ \\
\hline DA 4 & ARG 425 & $-1.0 \pm 1.8$ & $-1.0 \pm 3.2$ \\
\hline DG 5 & LYS 419 & $-7.8 \pm 8.3^{\mathrm{a}}$ & $-4.6 \pm 9.0^{\circ}$ \\
\hline DG 5 & ARG 425 & $-3.4 \pm 4.2$ & $-2.5 \pm 7.4$ \\
\hline DZ 6 & LYS 419 & $-1.3 \pm 1.9$ & $-0.5 \pm 1.8$ \\
\hline DZ 6 & ARG 425 & $-8.0 \pm 3.9^{\mathrm{a}}$ & $-3.3 \pm 3.4$ \\
\hline DG 7 & ARG 425 & $-1.8 \pm 2.6^{\mathrm{a}}$ & $-3.9 \pm 8.2^{2}$ \\
\hline $\mathrm{DC}^{\prime} 7$ & ARG 415 & $-3.1 \pm 8.8$ & $-0.8 \pm 4.4$ \\
\hline $\mathrm{DC}^{\prime} 7$ & MET 419 & $-1.3 \pm 1.5$ & $-0.4 \pm 0.8$ \\
\hline DC'7 & ASP 422 & $-3.6 \pm 4.4$ & $-4.0 \pm 2.3$ \\
\hline DC'7 & ARG 423 & $-1.0 \pm 3.5$ & $-0.3 \pm 1.8$ \\
\hline DA'9 & ARG 423 & $-2.0 \pm 3.1$ & $-1.7 \pm 8.4$ \\
\hline DA'8 & ARG 415 & $-2.3 \pm 2.5$ & $-1.9 \pm 5.0$ \\
\hline $\mathrm{DA}^{\prime} 8$ & ARG 423 & $-8.7 \pm 3.9^{\mathrm{a}}$ & $-5.4 \pm 8.9^{2}$ \\
\hline
\end{tabular}

${ }^{\text {a }}$ Most significant changes

O6 of 8-oxoguanine are found in less than $40 \%$ of the conformations considered. The presence of the oxidized guanine molecule forces increased mobility onto the arginine amino groups, which also confirms the values describing interactions with neighboring guanine molecules. For both amino groups of arginine, a significant increase in the number of hydrogen bonds formed with oxygen O6 (45\%) and nitrogen N7 (30\%) of guanine DG7 is observed. Interactions of arginine ARG425 recognizing oxidized CTT were also confirmed by RMSD values (Table 3).

In the S2 system, the RMSD and standard deviation values characterizing ARG425 are sevenfold higher than in S1. Thus, it is quite clear that oxidation of the central guanine molecule disturbing interactions inside the major groove is the source of the increased flexibility of this part of the protein chain. Consequently, interactions between lysine LYS 421 and the phosphate groups of nucleotides DA4 and DG5 are also affected by the presence of 8oxoG. Figure $4 \mathrm{~b}$ presents the distribution of distances between the two oxygen atoms found in the phosphate groups and the nitrogen atom on the protonated amino group. These two atoms were chosen because rotation of the tetragonal amino group interchanges the hydrogen atoms involved in H-bond formation. Analysis of these populations clearly identifies important differences in lysine interactions with native and oxidized telomeric B-DNA. In the case of the S1 system, both distributions describing binding between lysine LYS 421 and phosphate groups have quite a uniform shape. The number of conformations capable of forming hydrogen bonds exceeds $80 \%$ for DA4 and reaches $70 \%$ for DG5; however, differences in the activity of phosphate groups in native and oxidized systems are quite substantial. In the case of oxygen from the DA4 nucleotide, the number of conformations able to form hydrogen bonds increases up to $95 \%$ in the S2 system, while the activity of oxygen from the second nucleotide (DG5) decreased to nearly $45 \%$. These values suggest that increased mobility of arginine ARG425 has an influence on lysine interactions; consequently, the RMSD values characterizing lysine binding are reduced (see Table 1).

The complementary strand of the telomeric sequence creates hydrogen bonds with the amino groups of arginine ARG 423 and ARG 415. These two amino acids are involved in the creation of binding in the vicinity of the DNA backbone. Figures $4 \mathrm{c}$ and $5 \mathrm{a}, \mathrm{b}$ present distributions describing the activity of these subunits. The amino groups of arginine ARG 415 in the native system create hydrogen bonds with oxygens from the phosphate group of the DC7' nucleotide; such interactions can be found in $45 \%$ of analyzed conformations. Values collected for the oxidized systems indicate that such bindings do not occur in S2 structure. A second arginine molecule-ARG 423 - creates hydrogen bonds in another part of the BDNA helix. The interactions of ARG 423 with oxygen atoms from the phosphate group of the DA8' nucleotide are presented in Figs. $4 \mathrm{c}$ and $5 \mathrm{a}$.

External localization of the amino groups of arginine against the DNA major groove allows interactions with both oxygen atoms from the phosphate group of the DA8' nucleotide. Analysis of the distributions presenting mutual orientation of active groups in the native system allows us to conclude that one of the amino groups creates hydrogen bonds with the oxygen $\mathrm{O} 2$ during the entire simulation; one-half of the considered conformations can also create hydrogen bonds with the second oxygen atom $\mathrm{O} 1$. The second amino group exhibits less activity in binding; in this case, only $50 \%$ of conformations has the possibility to create hydrogen bonds with oxygens. The binding activity of arginine ARG 423 is decreased significantly in the oxidized system. The number of hydrogen bonds formed by both amino groups decreased by a factor of two in comparison with systems not containing the 8oxoguanine molecule. In all cases considered, oxidation was an important factor, changing the distribution of hydrogen bonds in the space encompassing the active site. Other interactions worth mentioning are those characterizing carboxyl groups from the side chain of aspartic acid ASP 422 interacting with hydrogen atoms from the amino group of cytosine DC7'. Analysis of these distributions (Fig. 5c) indicates that, in both native and oxidized systems, the frequencies of hydrogen bond creation are quite similar; however, in the S2 system, a slight decrease $(\sim 10 \%)$ in the number of conformations stabilized by this bond was observed.

These reported changes in hydrogen bonding and amino acid mobilities must also have an impact on hydrophobic 

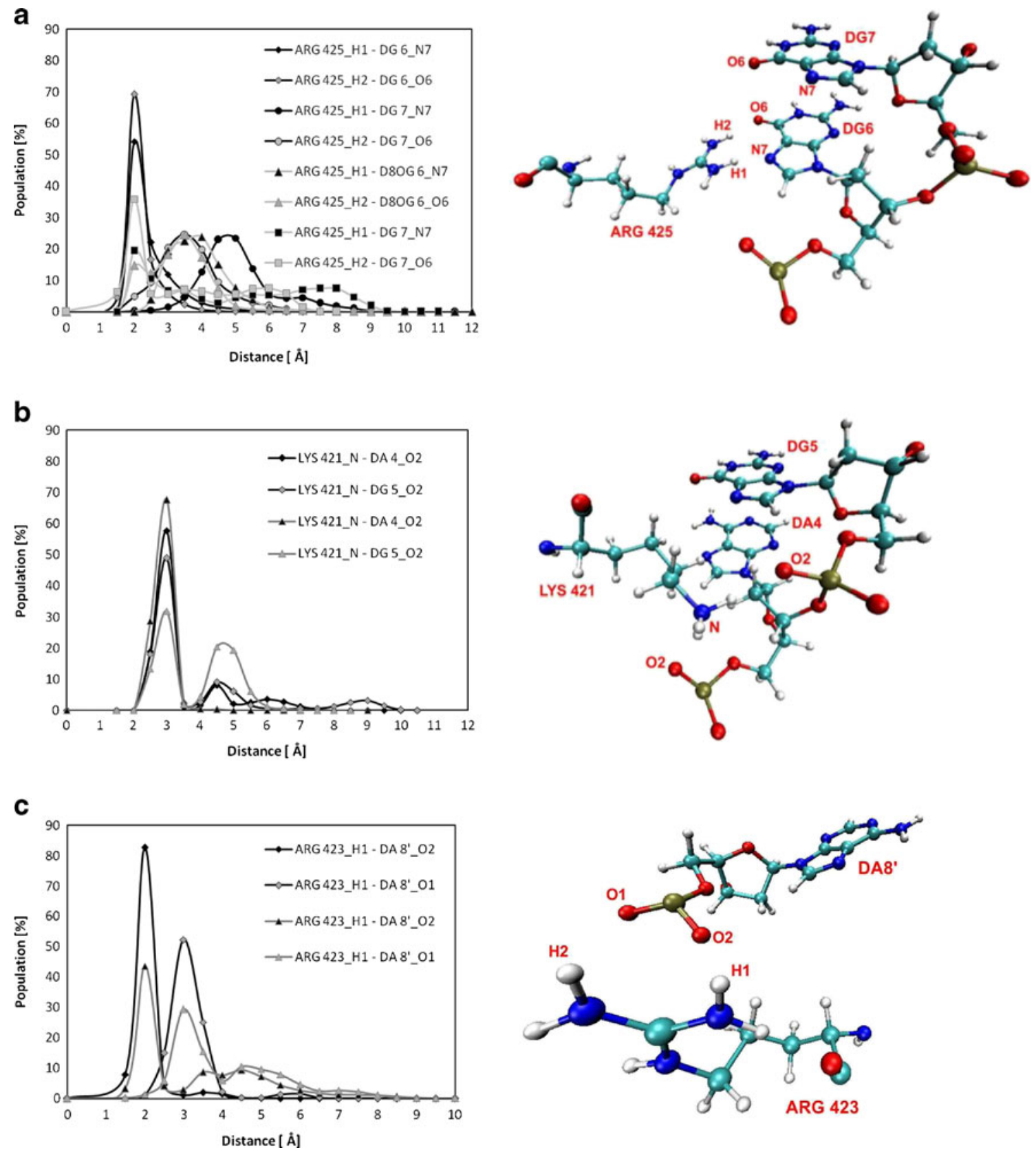

Fig. 4 Distributions of distances between groups of TRF1 and telomeric B-DNA involved in direct interactions. Black lines Native structure S1, gray lines oxidized systems $\mathrm{S} 2$

interactions. This all type of force contributing to the stability of telomeric complexes was analyzed by evaluation of the mutual orientation of selected structural groups from proteins and B-DNA. Figure 6 presents the distributions describing distances between carbon atoms and the considered monomers. Each case exhibits important differences between native
Table 3 Mean values of root mean square deviation (RMSD) and corresponding values of standard deviation describing the mobility of amino acids in the space of the major groove

\begin{tabular}{|c|c|c|c|c|c|c|}
\hline & \multicolumn{6}{|l|}{$\operatorname{RMSD}(\AA)$} \\
\hline & Arg 425 & Arg423 & Asp422 & Lys 421 & Met419 & Arg415 \\
\hline $\mathrm{S} 1$ & $0.18 \pm 0.08$ & $0.71 \pm 0.44$ & $0.80 \pm 0.42$ & $0.89 \pm 0.55$ & $1.55 \pm 0.88$ & $2.50 \pm 1.39$ \\
\hline $\mathrm{S} 2$ & $1.28 \pm 0.51$ & $0.83 \pm 0.31$ & $1.22 \pm 0.33$ & $1.31 \pm 0.40$ & $1.06 \pm 0.38$ & $1.49 \pm 0.56$ \\
\hline
\end{tabular}



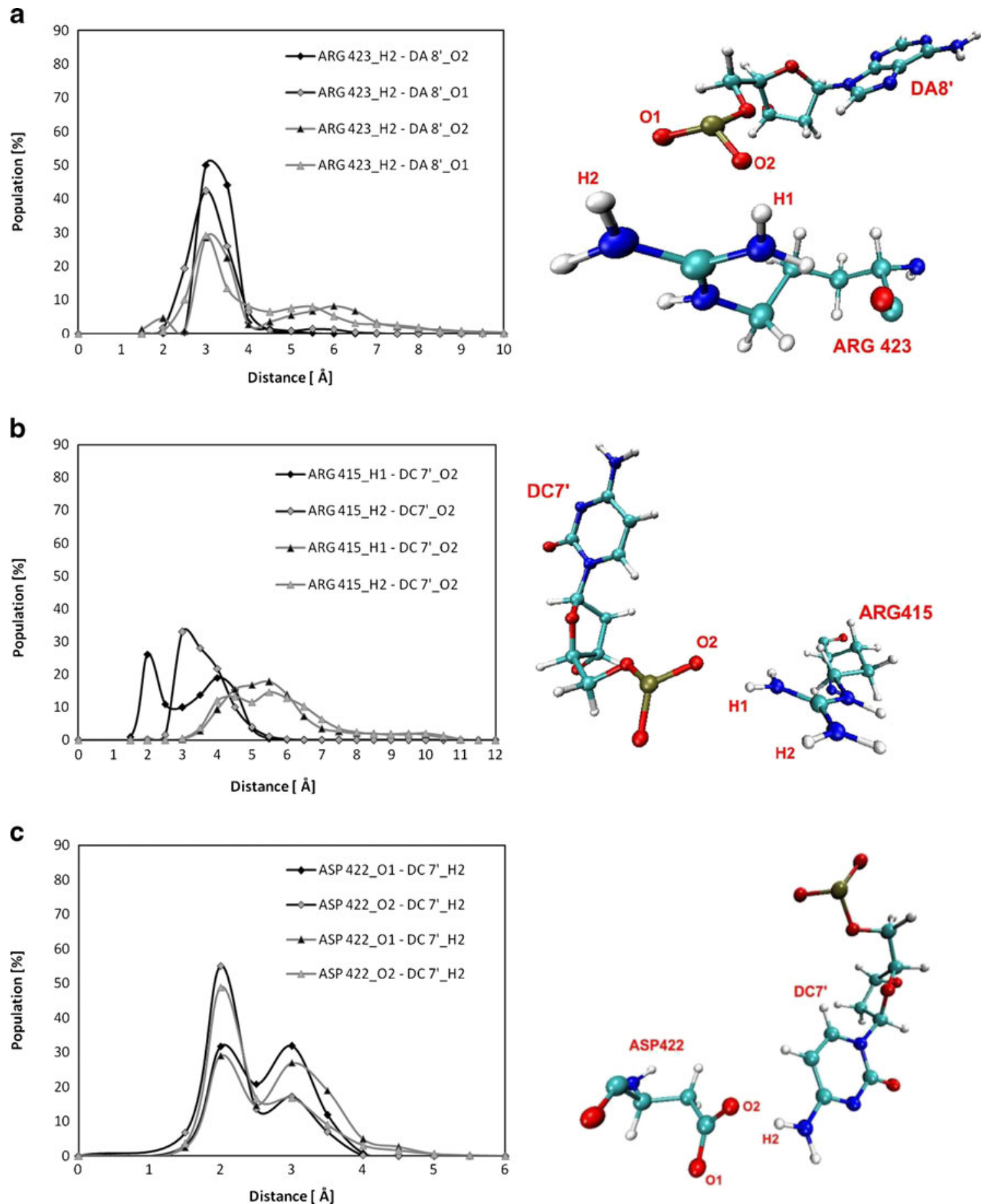

Fig. 5 Distributions of distances between groups of TRF1 and telomeric B-DNA involved in direct interactions. Black lines Native structure S1, gray lines oxidized systems S2

and oxidized systems. The largest differences are observed for methionine MET 419 interacting with the DC7' nucleotide. The values presented in Fig. 6a reveal the completely different behavior of the side chain of the considered amino acid. In the native system, distances between the methyl group of methionine and the carbons of deoxyribose and cytosine confirm the presence of non-negligible hydrophobic interactions in this region. On the contrary, in the oxidized system, distances between the considered groups increased two-fold in comparison to the reference system, suggesting much less effective interactions of the hydrophobic type.

Valine VAL 419 could also play an important role in the structure of these hydrophobic clusters. As revealed by the populations presented in Fig. 6b, methyl groups interact 

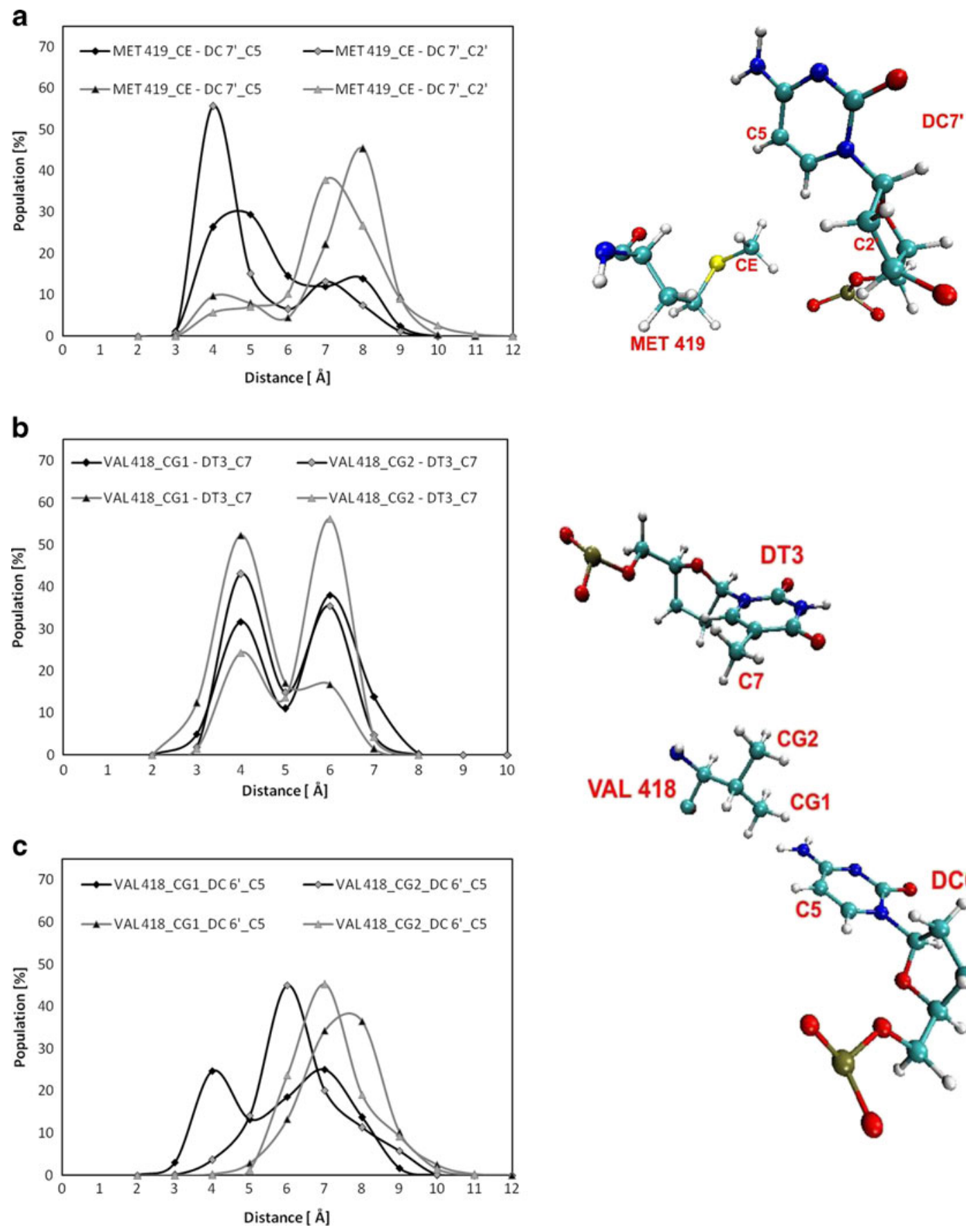

Fig. 6 Distributions of distances between hydrophobic groups of selected nucleic bases and amino acids localized in the space of the major groove. Black lines Native structure S1, gray lines oxidized

with both strands of B-DNA. The data suggest that, in both systems, mutual orientation of the side chain of valine and the methyl group of thymine DT3 allows the occurrence of hydrophobic interactions during most of the simulation time. Both methyl groups in the native system have quite a uniform distribution, while in the S2 system each methyl group is characterized by different values. Thus, only one systems S2. Measured values correspond to the distances between carbon atoms in the analyzed groups

methyl group is responsible for interactions with thymine DT3. Consequently, the mobility of valine is higher in the native system than in oxidized systems. Hydrophobic interactions of valine with cytosine DC6 ${ }^{\prime}$ on the complementary strand are less stable than those reported for thymine. In Fig. $6 c$, it can be seen that the distances between hydrophobicity centers increase in the oxidized systems compared to 
the reference system. All observed local structural alterations related both to hydrogen bonding interactions and hydrophobic cluster formation seriously influence the stability of the oxidized telomeric complex. The RMSD values presented in Fig. 7 unequivocally confirm the large structural and dynamic differences between the compared systems. All values describing the native telomeric complex (S1) and its subunits have quite uniform and narrow distributions. This suggests not only equilibration but also moderate flexibility of the native system. In contrast, systems comprising oxidized

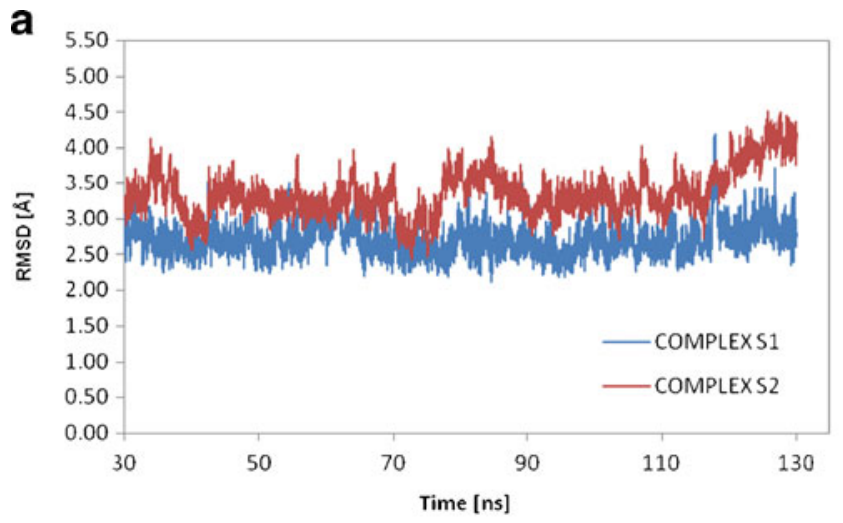

b

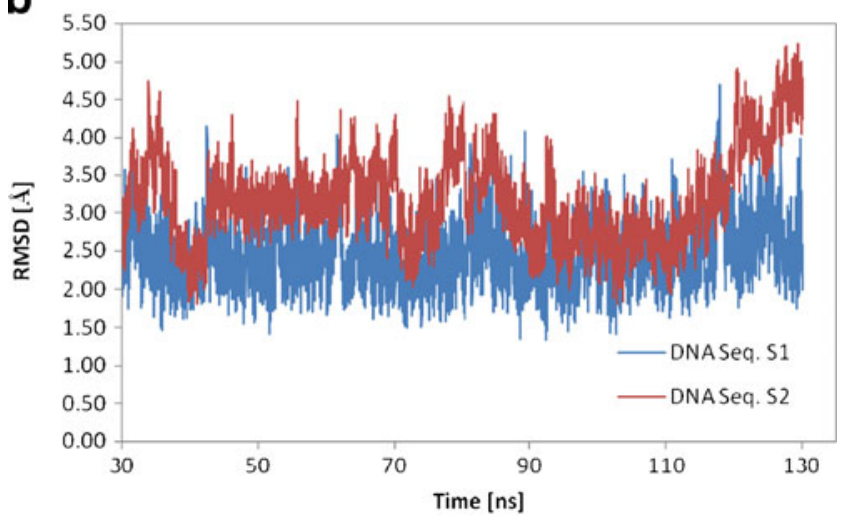

C

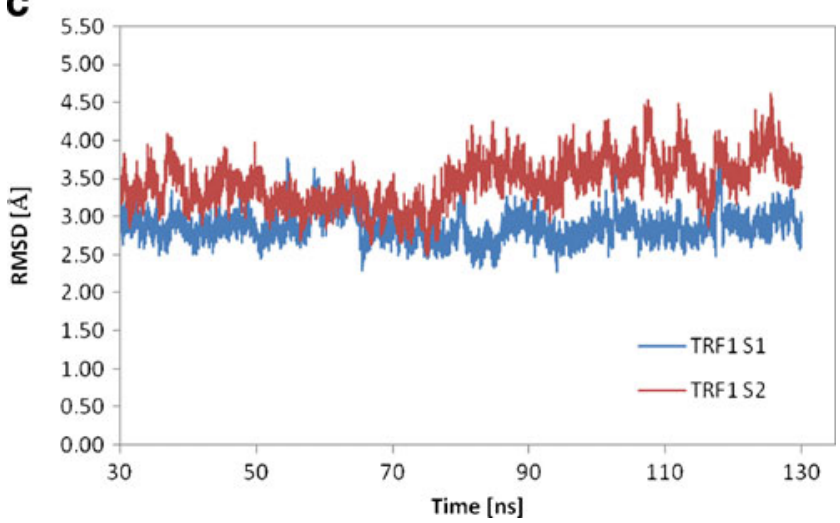

Fig. 7 RMSD values $[\AA]$ calculated for native (S1) and oxidized (S2) structures used during structural analysis. a Telomeric complex, b telomeric B-DNA sequence, $\mathbf{c}$ TRF1 protein ligand. All values were determined relative to the initial structure guanine (S2) are characterized by higher RMSD values. This indicates much more pronounced flexibility compared to the standard DNA-TRF1 complex. Furthermore, occasional sizable fluctuations of the S2 system are observed, suggesting structural disorders potentially imposed by the presence of the 8-oxoguanine molecule. Thus, weakening of TRF1-B-DNA complex stability originates from both structural and energetic consequences of telomere oxidation.

\section{Conclusions}

Complexes formed by the B-DNA telomeric subunit bound to TRF1 protein are very sensitive to the presence of 8-oxoguanine in the telomeric sequence. The data presented, characterizing both energetic and structural properties of the analyzed systems, indicate possible factors that are responsible for the weakening of complex stability after CTT oxidation. Guanine oxidation in the central telomeric fragment had both energetic and structural consequences. In particular, interactions of arginine ARG 425 with the guanine triad are crucial in the context of the dynamic properties of the complete C-terminal protein helix of the TRF1 ligand. The decrease in binding affinity of ARG 425 - DZ6 subunits, and the accompanying increase in binding between ARG 425 and DG7 observed in oxidized systems correlates with changes in the frequency of hydrogen bond formation between the considered monomers. The origin of increased mobility of arginine ARG 425 and its movement in the space of the major groove is the alternate donor-acceptor pattern corresponding to replacement of guanine with 8-oxoguanine. The hydrogen atom connected to the capping nitrogen N7 significantly hampers interactions with proton-accepting amino-groups of the selected amino acid. It is worth mentioning that changes in the interactions between arginine and the guanine triad are also accompanied by discrepancies in the behavior of other monomers residing in the $\mathrm{C}$-terminal protein helix. The significant mobility of lysine belonging to a side chain along the main strand of B-DNA influences the energetics of interactions with DA4 and DG5 nucleotides. Similar relationships are also observed in the complementary strand due to interactions of arginine ARG 415 with DC7' and ARG 423 with the DA8' nucleotide. Furthermore, hydrophobic interactions also make an important contribution to the stabilization of the analyzed systems. Despite the lack of direct interaction between the 8-oxoguanine molecule and amino acids, significant changes in the properties of the hydrophobic clusters formed in the space of major groove were observed. The large increase in distance and changes in the mobility of monomers involved in creation of hydrophobic interactions certainly affects their efficiency. All the above observations lead to the conclusion that oxidation of the central guanine molecule in CTT is the main source of both the global and local 
energetic and structural changes that are responsible for significant weakening of TRF1 affinity for telomeric B-DNA complex formation.

Acknowledgments The results presented here were obtained as part of computational grant no 104 of the Poznan Supercomputing and Networking Center (Poznań, Poland) with facilities provided by the Academic Computer Centre CYFRONET within PLGrid infrastructure. The allocation of computational facilities is greatly appreciated.

Open Access This article is distributed under the terms of the Creative Commons Attribution License which permits any use, distribution, and reproduction in any medium, provided the original author(s) and the source are credited.

\section{References}

1. Chong L, van Steensel B, Broccoli D, Erdjument-Bromage H, Hanish J, Tempst P, de Lange T (1995) A human telomeric protein. Science 270:1663-1667

2. Cook DB, Dynet JN, Chang W, Shostak G, Smith S (2002) Role for the related polyADP-ribose polymerases tankyrase 1 and 2 at human telomeres. Mol Cell Biol 22:332-342

3. Li B, Oestreich S, de Lange T (2000) Identification of human Rap1: implications for telomere evolution. Cell 101:471-483

4. Kim SH, Kaminker P, Campisi J (1999) TIN2, a new regulator of telomere length in human cells. Nat Genet 23:405-412

5. Smith S, Giriat I, Schmitt A, de Lange T (1998) Tankyrase, a poly (ADP-ribose) polymerase at human telomeres. Science 282:14841487

6. Griffith JD, Comeau L, Rosenfiel S, Stansel RM, Bianchi A, Moss $\mathrm{H}$, de Lange T (1999) Mammalian telomeres end in a large duplex loop. Cell 97:503-514

7. de Lange T (2005) Shelterin: the protein complex that shapes and safe-guards human telomeres. Genes Dev 19:2100-2110

8. de Lange T (2004) $T$-loops and the origin of telomeres. Nat Rev Mol Cell Biol 5:323-329

9. Fairall L, Chapman L, Moss H, de Lange T, Rhodes D (2001) Mol Cell 8:351-361

10. De Lange T (2002) Protection of mammalian telomeres. Oncogene 21:532-540

11. Lundblad V (2000) DNA ends: maintenance of chromosome termini versus repair of double strand breaks. Mutat Res 451:227-240

12. Harley CB, Futcher AB, Greider CW (1990) Telomeres shorten during ageing of human fibroblasts. Nature 345:458-460

13. von Zglinicki T, Serra V, Lorenz M, Saretzki G, LenzenGrossimlighaus R, Gressner R, Risch Steinhagen-Thiessen E (2000) Short telomeres in patients with vascular dementia: an indicator of low antioxidative capacity and a possible risk factor? Lab Invest 80:1739-1747

14. Bodnar AG, Ouellette M, Frolkis M, Holt SE, Chiu CP, Morin GB, Harley CB, Shay JW, Lichtsteiner S, Wright WE (1998) Extension of life-span by introduction of telomerase into normal human cells. Science 279:349-352

15. Blackburn EH (2001) Switching and signaling at the telomere. Cell 106:661-673

16. Cervantes RB, Lundblad V (2002) Mechanisms of chromosomeend protection. Curr Opin Cell Biol 14:351-356

17. Chan SWL, Blackburn EH (2002) New ways not to make ends meet. Oncogene 21:553-563

18. McEachern MJ, Krauskopf A, Blackburn EH (2000) Telomeres and their control. Annu Rev Genet 34:331-358
19. Zakian VA (1995) Telomeres: beginning to understand the end. Science 270:1601-1607

20. Moyzis RK, Buckingham JM, Cram LS, Dani M, Deaven LL, Jones MD, Meyne J, Ratliff RL, Wu JR (1988) A highly conserved repetitive DNA sequence (TTAGGG) , present at the telomeres of human chromosomes. Proc Natl Acad Sci USA 85:6622-6626

21. Petersen S, Saretzki G, von Zglinicki T (1998) Preferential accumulation of single-stranded regions in telomeres of human fibroblasts. Exp Cell Res 239:152-160

22. Armitage B (1998) Photocleavage of nucleic acids. Chem Rev 98:1171-1200

23. Barrows CJ, Muller JG (1998) Oxidative nucleobase modifications leading to strand scission. Chem Rev 98:1109-1151

24. Cysewski P (2008) The impact of the nucleoside oxidation on the susceptibility to chemical carcinogens studied by first principle and semiempirical quantum chemistry methods. THEOCHEM 863:16-21

25. Meggers E, Michel-Beyerle ME, Giese B (1998) Sequence dependent long range transport in DNA. J Am Chem Soc 120:12950-12955

26. Giese B (2000) Long distance charge transport in DNA: the hopping mechanism. Acc Chem Res 33:631-636

27. Sugiyama H, Saito I (1996) Theoretical studies of GG-specific photocleavage of DNA via electron transfer: significant lowering of ionization potential and 5'-localization of HOMO of stacked GG bases in B form DNA. J Am Chem Soc 118:7063-7068

28. Cysewski P, Czeleń P (2010) Structural and energetic consequences of oxidation of $\mathrm{d}(\mathrm{ApGpGpGpTpT)}$ telomere repeat unit in complex with TRF1 protein. J Mol Model 16:1797-1807

29. Cysewski P, Czeleń P (2009) Structural and energetic heterogeneities of canonical and oxidized central guanine triad of B-DNA telomeric fragments. J Mol Model 15:607-613

30. Tchirkov A, Lansdorp PM (2003) Role of oxidative stress in telomere shortening in cultured fibroblasts from normal individuals and patients with ataxia-telangiectasia. Hum Mol Genet 12:227-232

31. von Zglinicki T (2002) Oxidative stress shortens telomeres. Trends Biochem Sci 27:339-344

32. Opresko PL, Fan J, Danzy S, Wilson DM 3rd, Bohr VA (2005) Oxidative damage in telomeric DNA disrupts recognition by TRF1 and TRF2. Nucleic Acids Res 33:1230-1239

33. Cysewski P, Czeleń P (2007) Theoretical analysis of the effects of guanine oxidative damage on the properties of B-DNA telomere fragments. J Mol Model 13:739-750

34. Court R, Chapman L, Fairall L, Rhodes D (2005) How the human telomeric proteins TRF1 and TRF2 recognize telomeric DNA: a view from high-resolution crystal structures. EMBO Rep 6:39-45

35. Hanaoka S, Nagadoi A, Nishimura Y (2005) Comparison between TRF2 and TRF1 of their telomeric DNA-bound structures and DNA-binding activities. Protein Sci 14:119-130

36. Nishikawa T, Okamura H, Nagadoi A, Konig P, Rhodes D, Nishimura Y (2001) Solution structure of the DNA complex of human TRF1. Structure 9:1237-1251

37. Jorgensen WL, Chandrasekhar J, Madura JD, Impey RW, Klein ML (1983) Comparison of simple potential functions for simulating liquid water. J Chem Phys 79:926-935

38. de Souza ON, Ornstein RL (1997) Effect of periodic box size on aqueous molecular dynamics simulation of a DNA dodecamer with particle-mesh Ewald method. Biophys J 72(6):2395-2397

39. Ryckaert JP, Ciccotti G, Berendsen HJC (1977) Numerical integration of the Cartesian equations of motion of a system with constraints: molecular dynamics of n-Alkanes. J Comput Phys 23 (3):327-341

40. Humphrey W, Dalke A, Schulten K (1996) VMD: visual molecular dynamics. J Mol Graph 14:33-38

41. Miller BR, McGee TD, Swails JM, Homeyer N, Gohlke H, Roitberg AE (2012) MMPBSA.py: an efficient program for end-state free energy calculations. J Chem Theory Comput 8(9):3314-3321 
42. Case DA, Darden TA, Cheatham TE III, Simmerling CL, Wang J, Duke RE, Luo R, WalkerRC ZW, Merz KM, Roberts B, Wang B, Hayik S, Roitberg A, Seabra G, Kolossváry I, Wong KF, Paesani F, Vanicek J, Liu J, Wu X, Brozell SR, Steinbrecher T, Gohlke H, Cai Q, Ye X, Wang J, Hsieh MJ, Cui G, Roe DR, Mathews DH, Seetin MG, Sagui C, Babin V, Luchko T, Gusarov S, Kovalenko A, Kollman PA (2010) AMBER 11. University of California, San Francisco
43. Pérez A, Marchán I, Svozil D, Šponer J, Cheatham TE III, Laughton CA, Orozco M (2007) Refinement of the AMBER force field for nucleic acids: improving the description of conformers. Biophys J 92:3817-3829

44. Miller JH, Fan-Chiang CP, Straatsma TP, Kennedy MA (2003) 8oxoguanine enhances bending of DNA that favors binding to glycosylases. J Am Chem Soc 125:6331-6336 